\title{
Reconstruction of carotid artery pseudoaneurysm in an intravenous drug user with multiple needles in the neck
}

\author{
Mihaly $Z^{1}$, Jermendy $A L^{1}$, Osztrogonacz $\mathrm{P}^{1}$, Nemes $\mathrm{B}^{1}$, Olah $Z^{1}$, Folyovich $\mathrm{A}^{2}$, Beres Molnar KA ${ }^{2}$, Szeberin $Z^{1}$ and Sotonyi $\mathrm{P}^{*}$ \\ ${ }^{1}$ Department of Vascular Surgery, Semmelweis University, Budapest, Hungary \\ ${ }^{2}$ Szent Janos Hospital, Department of Neurology and Stroke Centre, Budapest Hungary
}

\begin{abstract}
Introduction: There are very few intravenous drug abuse-related carotid pseudoaneurysm cases reported in the literature. We report a case of a carotid pseudoaneurysm reconstruction with vascular allograft due to recurrent infection caused by frequent cervical self-punctions, and a subsequent recurrent septic pseudoaneurysm formation.

Case report: A 45-year-old iv. heroin user male patient was admitted to our department. The left cervical region became swollen causing dyspnea and showed pulsation. The CT angiography revealed broken needles in both cervical regions and a left common carotid artery pseudoaneurysm with a diameter of $5 \mathrm{~cm}$ that caused dislocation of the trachea. We performed an urgent resection of the pseudoaneurysm and reconstruction with a cryopreserved human arterial allograft. Two months later the patient returned to our unit with dyspnea, dizziness, fever and a pulsating cervical mass at the operative site following repeated drug abuse, and the direct puncture of the vascular allograft. The ultrasound examination showed a contained rupture at the proximal anastomosis. During his preparation for the urgent surgery he suffered a stroke causing right hemiplegia and aphasia. We occluded the common carotid artery with an Amplatzer vascular plug and the hematoma was evacuated.

Discussion: Cervical foreign bodies rarely cause recurrent carotid pseudoaneurysm. An endovascular occlusion of the common carotid artery was necessary for anastomotic disruption of allograft placed for a common carotid pseudoaneurysm.

Conclusion: Patients with infected carotid artery pseudoaneurysm should be treated by open or endovascular methods to prevent stroke or any life-threatening complications.
\end{abstract}

\section{Introduction}

Extracranial carotid artery aneurysms (ECAA) are uncommon, contributing to less than $1 \%$ of all arterial aneurysms $[1,2]$. The most common causes of ECAA are atherosclerosis, trauma, fibromuscular dysplasia, previous surgery, congenital defects, infection and irradiation. Most of the carotid artery aneurysms are pseudoaneurysms, which can manifest with varying symptoms [3]. Incidence of mycotic pseudoaneurysms is low, being reported in less than $5 \%$ of all cases of arterial pseudoaneurysms [4]. According to Tsao and his colleagues, intravenous drug use was the etiology in $0.03 \%$ of all mycotic aneurysms [5]. Currently, there is no level IA recommendation for the treatment of ECAA cases. Only a small number of case reports and reviews can be found in the literature covering this topic. Open surgical reconstruction has been the first-line treatment of ECAA, even with the increasing availability of endovascular capabilities. The five basic options for open surgical intervention - aneurysm clipping, excision with primary anastomosis, excision with graft interposition, extracranial-intracranial bypass and carotid ligation - were sorted by Garg and his colleagues [6]. Open surgical intervention was favored when symptoms were present, especially mass effect or bleeding. Open repair is frequently criticized for its high rate of cranial nerve deficit, occurring in $2.6-12 \%$ of all cases $[3,7]$. Technical limitations of open repair may emerge when the aneurysm is located near to the skull base and in patients with hostile neck due to previous neck surgery or irradiation.

Although the number of the intravenous drug users in Hungary is low, there are some cases of needle breakage every year at the vascular surgery departments. Needle fragments in the neck are unlikely to result late complications according to the study Williams et al. based on 50 patients, therefore their removal was not recommended by the authors [8]. The most common incidence of cervical foreign bodies is in older iv. drug users without any applicable peripheral veins. Williams's review summarized the most common complications of neck self-injection: infections, hematoma, venous thrombosis, vocal cord paralysis. Among their cases there were no delayed complications.

\section{Case report}

A 45-year-old intravenous heroin user male patient was admitted to our department. He used to inject heroin with insulin syringes directly into the jugular vein or common carotid artery in the supraclavicular region on both sides. Three weeks before admission, he had fallen, and his left cervical region begun to swell. He was admitted to a hospital but was soon discharged because of the lack of need for urgent intervention. His symptoms progressed and eventually dyspnea and fever presented. Upon admission to our institution, the laboratory results showed elevated inflammatory markers (WBC: $16.3 \mathrm{G} / \mathrm{l}$, CRP: $178.3 \mathrm{mg} / \mathrm{l})$. In the cervical region there were no signs

${ }^{*}$ Correspondence to: Peter Sotonyi, Department of Vascular Surgery, Semmelweis University, Budapest, Hungary, H-1122 Budapest, Varosmajor str 68, E-mail: sotonyi@hotmail.com

Key words: common carotid artery pseudoaneurysm, iv. drug abuse, neck foreign body, cryopreserved human arterial allograft

Received: June 15, 2019; Accepted: July 11, 2019; Published: July 15, 2019 
of local infection, however, extensive cutaneous scarring was visible (Figure 1A). Echocardiography excluded endocarditis; hepatitis and HIV serology tests were negative. The CT angiography (CTA) showed broken needles in both cervical regions and a pseudoaneurysm of the left common carotid artery with a diameter of $5 \mathrm{~cm}$ causing tracheal dislocation (Figure 1B). Urgent surgery was performed in general anesthesia. The incision was made anterior to the sternocleidomastoid muscle. The dissection was challenging due to chronic scar tissues. It was neither possible to find nor to extract the needle fragments. Before the pseudoaneurysm was exposed, 10.000 I.U. of Heparin were administered iv. and the common, external and internal carotid arteries were clamped. After the resection of the pseudoaneurysm, the distal part of the common carotid artery and the internal carotid artery was replaced with a cryopreserved superficial femoral artery graft with end-to-end anastomoses (Figure 2A and 2B). The external carotid and the superior thyroid arteries were ligated. Microbiological samples were taken from the surgical site. During the postoperative care, no neurological symptoms developed and there was no sign of peripheral nerve injury. The intraoperative microbiological samples were all negative, and the inflammatory markers decreased (WBC: $10.12 \mathrm{G} / \mathrm{l}$, CRP: $21.33 \mathrm{mg} / \mathrm{l})$. Oral ciprofloxacin and clindamycin were administered.

Two months later the patient was readmitted due to fever and dizziness, following repeated drug abuse, and the direct puncture of the vascular allograft. The US examination showed a large pseudoaneurysm in the proximal anastomosis of the allograft. The CTA revealed a new needle fragment crossing the interposition, proximal anastomosis pseudoaneurysm and cervical hematoma. The blood culture indicated Staphylococcus aureus infection, therefore iv. cephalosporin therapy was initiated. Soon after his admission, the patient developed right hemiplegia and aphasia. Repeated CTA (Figure 3A and 3B) showed acute intracranial internal carotid occlusion and extensive ischemic lesion in the area of the left middle cerebral artery (Aspect score: 5). In order to prevent pseudoaneurysm rupture leading to fatal bleeding, we occluded the common carotid artery with an Amplatzer II plug (12 $\mathrm{mm}$; St. Jude Medical, Inc., St. Paul, MN) via a femoral puncture. An incision was made on the cervical mass to evacuate the hematoma and to prevent cervical phlegmone. The secondary intention wound healing
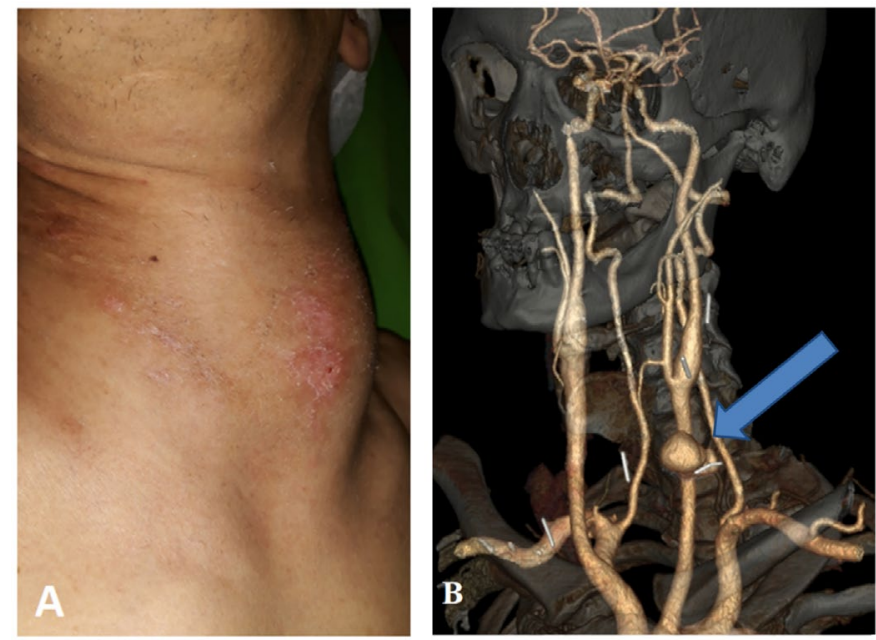

Figure 1. Figure A shows the pulsating mass in the cervical region. Figure B: the white lines are the needle fragments, and the arrow shows the pseudoaneurysm of the common carotid artery on the CT angiography
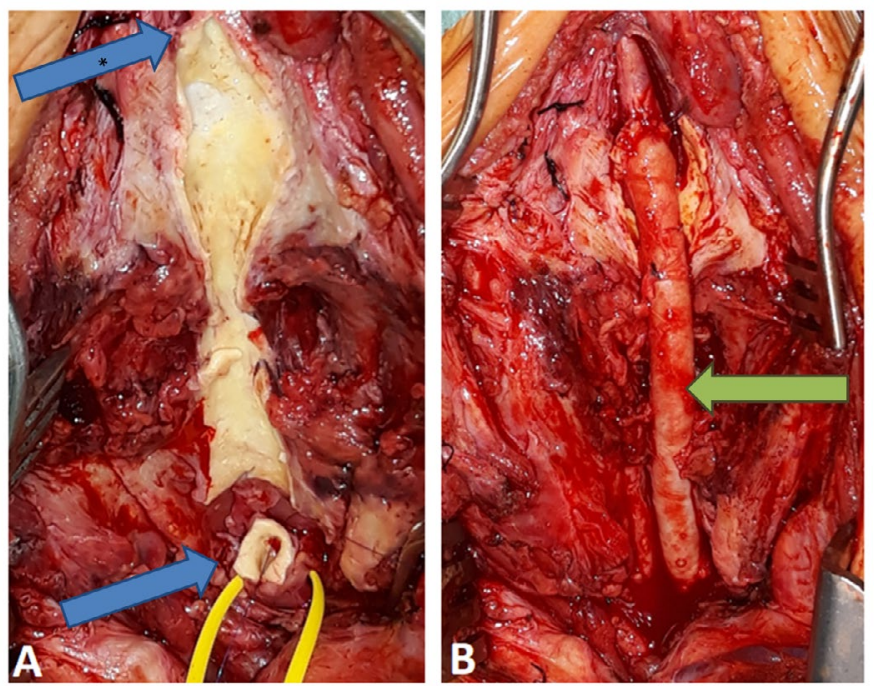

Figure 2. Figure A shows the remaining posterior wall of the resected pseudoaneurysm, the blue arrow with asterisk indicates the internal carotid artery. The blue arrow indicates the proximal common carotid artery. Figure B: the green arrow indicates the cryopreserved human arterial allograft
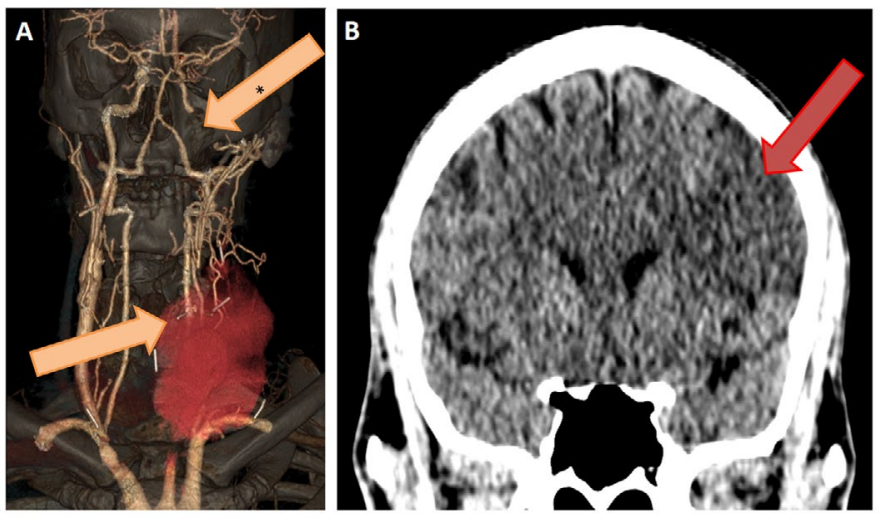

Figure 3. Figure A: the orange arrow with asterisk indicates the occluded distal interna carotid artery. The orange arrow shows the new needle fragment crossing the allograft interposition, proximal anastomosis pseudoaneurysm and cervical hematoma on CT angiograpy. Figure B: there is an extensive ischemic lesion in the area of the left middle cerebral artery on cranial CT

was prolonged. The patient's neurological function slowly improved due to the intensive rehabilitation program.

\section{Discussion}

Carotid artery pseudoaneurysms caused in drug abusers by iv. needle injuries are rare. According to a review by Fankhauser et al., only half of the 116 patients with carotid pseudoaneurysms had any symptoms and there were no intravenous drug users in this cohort [9]. Another meta-analysis of 392 patients with ECAAs mentioned traumatic pseudoaneurysms in $14 \%$ [2]. In the meta-analysis of endovascular ECAA reconstructions, there were only 6 cases with infection and there was no mention of intravenous drug use as an etiology either, despite the large number of cases (211 pseudoaneurysms) [1].

A case review with 30 mycotic ECAAs suggests that resection of the aneurysm and reconstruction of the arterial supply to the brain by graft interposition is the preferred procedure along with longterm antibiotic treatment. Prosthetic grafts should be avoided to prevent graft infection [10]. The use of endovascular techniques still 
remains controversial in cases with infection, although it can be an attractive alternative in the case of high-risk patients or in instances of a challenging surgical approach [11]. There are limited experiences with hybrid reconstruction of carotid patch infection with endo-VAC treatment [12]

There is one iv. drug abuse related case reported by Teebken et al., an urgent carotid reconstruction was performed with a homograft patch repair [13]. An Italian group presented a case of a hybrid reconstruction of an infected right internal carotid artery pseudoaneurysm, where the pseudoaneurysm was bridged with a covered stent, before a right side laterocervical surgical incision was performed. The hematoma was evacuated, and a PTFE graft was wrapped around the carotid wall where the covered stent had been placed [14]. In another case report of a patient with massive epistaxis, the cause of the intracranial left internal carotid artery pseudoaneurysm was an intranasal foreign object without apparent recent trauma. The patient was successfully treated with endovascular stenting of the affected vessel segment [15]. An international carotid registry may yield growing knowledge how to treat this rare condition [3].

In our case, a carotid artery pseudoaneurysm developed in an intravenous drug user due to late complication of direct neck punctures. Metal foreign bodies as previously inserted and fragmented needles are well described in iv. drug users, and their complications are mostly prompt but late complications are rare [8]. In this case a late complication of cervical foreign bodies was urgently operated, the foreign bodies were irremovable from the scar tissue during the intervention without further risk of nerve or blood vessel injury. A homologous graft was used for the interposition, as although microbiological samples turned out to be negative, we had to consider the site infected, especially in view of the chronic foreign bodies. At the second admission open reconstruction was determined to carry extreme high risk due to the possibility of uncontrolled bleeding, therefore this solution was rejected. The new onset neurological event and the internal carotid artery occlusion ruled out covered stent implantation. The Amplatzer vascular plug implantation was a safe option for carotid artery occlusion, although there was a risk of bloodstream infection. The hematoma evacuation was obligatory due to the skin necrosis and in order to remove the potential source of infection.

\section{Conclusion}

Patients with carotid pseudoaneurysms caused by cervical foreign bodies should be urgently admitted to specialized hospitals in case of neck swelling, dyspnea, or dysphagia. CT or MR should be performed in order to plan the intervention and avoid fatal airway compression, bleeding or stroke. Open and endovascular surgical methods can be used to save the patients' life in this rare situation.

\section{Conflicts of interest}

\section{None}

\section{References}

1. Li Z, Chang G, Yao C (2011) Endovascular stenting of extracranial carotid artery aneurysm: a systematic review. Eur J Vasc Endovasc Surg 42: 419-426

2. El-Sabrout R, Cooley DA (2000) Extracranial carotid artery aneurysms: Texas Heart Institute experience. J Vasc Surg 31: 702-712

3. Welleweerd JC, den Ruijter HM, Nelissen BG, Bots ML, Kappelle LJ, et al (2015) Management of extracranial carotid artery aneurysm. Eur J Vasc Endovasc Surg 50:141-147.

4. Brown SL, Busuttil RW, Baker JD, Machleder HI, Moore WS, et al. (1984) Bacteriologic and surgical determinants of survival in patients with mycotic aneurysms. J Vasc Surg 1: 541-547

5. Tsao JW, Marder SR, Goldstone J, Bloom AI (2002) Presentation, diagnosis, and management of arterial mycotic pseudoaneurysms in injection drug users. Ann Vasc Surg 16: 652-662.

6. Garg K, Rockman CB, Lee V, Maldonado TS, Jacobowitz GR, Adelman MA. et al. (2012) Presentation and management of carotid artery aneurysms and pseudoaneurysms. J Vasc Surg 55: 1618-1622

7. Attigah N, Kulkens S, Zausig N, Hansmann J, Ringleb P et al. (2009) Surgical therapy of extracranial carotid artery aneurysms: long-term results over a 24-year period. Eur $J$ Vasc Endovasc Surg 37: 127-133.

8. Williams MF, Eisele DW, Wyatt SH (1993) Neck needle foreign bodies in intravenous drug abusers. Laryngoscope 103: 59-63.

9. Fankhauser GT, Stone WM, Fowl RJ, O'Donnell ME, Bower TC, et al. (2015) Surgical and medical management of extracranial carotid artery aneurysms. J Vasc Surg 61: 389-393.

10. Pirvu A, Bouchet C, Garibotti FM, Haupert S, Sessa C, et al. (2013) Mycotic aneurysm of the internal carotid artery. Ann Vasc Surg 27: 826-830.

11. Baril DT, Ellozy SH, Carroccio A, Patel AB, Lookstein RA, Marin ML, et al. (2004) Endovascular repair of an infected carotid artery pseudoaneurysm. J Vasc Surg 40: $1024-1027$

12. Thorbjornsen K, Djavani Gidlund K, Bjorck M (2016) Long-term Outcome After EndoVAC Hybrid Repair of Infected Vascular Reconstructions. Eur J Vasc Endovasc Surg 51: 724-732.

13. Teebken OE, Pichlmaier M, Leinung M, Lenarz T, Haverich A, et al. (2007) Homograf patch repair in carotid artery rupture. Vasa 36: 279-281.

14. Mazzaccaro D, Stegher S. Occhiuto MT, Malacrida G, Righini P, Tealdi DG, et al. (2014) Hybrid endovascular and surgical approach for mycotic pseudoaneurysms of the extracranialinternal carotid artery. SAGE Open Med Case Rep 19: 2-2050313X14558081

15. Katsevman GA, Braca JA, Welch KC, Ashley WW (2016) Delayed presentation of an extracranial internal carotid artery pseudoaneurysm and massive epistaxis secondary to a nasal foreign body: Case report and review of the literature. World Neurosurg 92 585.e13-585

Copyright: (C2019 Mihaly Z. This is an open-access article distributed under the terms of the Creative Commons Attribution License, which permits unrestricted use, distribution, and reproduction in any medium, provided the original author and source are credited. 